\title{
Experiencia con un método de autotoma de muestra vaginal para la detección de infección por Chlamydia trachomatis y Neisseria gonorrhoeae en mujeres jóvenes
}

\author{
Carolina Conejero, Gigliola Cannoni, Paulina M. Merino, Josefina Bollmann, Camila Hidalgo, \\ Magdalena Castro y Carolina Schulin-Zeuthen
}

\section{Screening of Neisseria gonorrhoeae and Chlamydia trachomatis using techniques of self collected vaginal sample in young women}

Introduction: Chlamydia trachomatis and Neisseria gonorrhoeae are responsible for 3-10\% of sexually transmitted diseases in adolescents. $75 \%$ are asymptomatic. International standards recommend annual screening for C. trachomatis in sexually active women under 26 years. Self-collected vaginal swab is one of the less invasive screening methods, it is well accepted by patients and rarely used in our country. Aim: To determine the frequency of $C$. trachomatis and $N$. gonorrhoeae by a self-sampling method of vaginal swab and its acceptability in a group of adolescents and young adults. Patients and Methods: Women 18 to 25 years old. Vaginal samples were processed by nucleic acid amplification tests, Gen Probe APTIMA Combo2. Data were collected on sexual behavior and perception of self-sampling by survey. Results: We studied 344 patients with an average age of 21.7 years. Detection of C. trachomatis was positive in 7.9\% women and it was not found in any of the patients studied for N. gonorrhoeae. $98 \%$ considered self-sampling instructions easy to understand, $87.5 \%$ felt comfortable taking the sample. Conclusions: Prevalence of C. trachomatis in the study population was similar to that described in other national and international studies. $N$. gonorrhoeae was not found in this series, which is consistent with literature reports. The self-sampling technique of vaginal sample was well accepted by the patients. However, they were anxious about the quality of the sample. According to our results, it is important to emphasize the importance of annual detection of these pathogens and that self-sampling technique is a valid alternative.

Key words: Chlamydia trachomatis, screening, self-collected vaginal swabs.

Palabras clave: Chlamydia trachomatis, tamizaje, autotoma vaginal.

\section{Introducción}

$\mathrm{L}$ as infecciones genito-urinarias por Chlamydia trachomatis y Neisseria gonorrhoeae son las infecciones bacterianas de transmisión sexual más frecuentes en el mundo, especialmente en el grupo de mujeres adolescentes y adultas jóvenes. Si bien, la mayoría de los casos de infección en mujeres son asintomáticos (hasta $75 \%$ de los casos) $)^{1}$, su mayor impacto proviene de las secuelas anatómicas secundarias al proceso inflamatorio asociado, que puede eventualmente afectar el futuro reproductivo de la paciente. Se estima que 2 a $10 \%$ de las infecciones por $C$. trachomatis podrían evolucionar a proceso inflamatorio pélvico (PIP) y $0,1-6 \%$ podrían presentar infertilidad por factor tubo-peritoneal ${ }^{2}$. Por otra parte, la infección por $C$. trachomatis sería un cofactor en el desarrollo inicial de neoplasia intraepitelial cérvico-uterina ${ }^{3} \mathrm{y}$ en la transmisión del VIH, si bien hasta ahora se desconocen los mecanismos subyacentes a estos procesos ${ }^{4}$.

La prevalencia de infección por C. trachomatis en países desarrollados varía entre 3 y $10 \% \%^{5-7}$. Estudios realizados en Latinoamérica reportan prevalencias ligeramente superiores. En Colombia se ha descrito una frecuencia cercana a 5\% $\%^{8,9}$, en Perú $6,8 \%{ }^{10}$, en Brasil entre $11,4 \%$ y $19 \%^{11,12}$ y en Venezuela $10,4 \%{ }^{13}$. En Chile existen pocos estudios al respecto. Martínez y cols. ${ }^{14}$, en un grupo de 403 mujeres con un promedio de edad de 34 años, comunicó una prevalencia de 4,7\% para infección por $C$. trachomatis. El único estudio realizado exclusivamente en adolescente y jóvenes chilenas, incluyó 203 pacientes entre 12 y 25 años; de ellas, 6,9\% fueron positivas para C. trachomatis ${ }^{15}$.

La prevalencia de $N$. gonorrhoeae reportada en Estados Unidos de América (E.U.A.) es de $0,43 \%$ en población adulta de ambos $\operatorname{sexos}^{5}$, siendo la segunda infección de transmisión sexual (ITS) más frecuentemente notificada. Otros estudios han demostrado una prevalencia de 0,08 a $0,42 \%{ }^{16}$. La incidencia general de $N$. gonorrhoeae en Chile ha ido descendiendo desde 12,8 por 100.000 habitantes en el año 2002 a 7,6 por 100.000 habitantes en el año $2007^{17}$.
Clínica Las Condes, Santiago, Chile.

Departamento de Obstetricia y Ginecología

Programa de Ginecología de la Adolescencia (CC, GC, PMM, CS-Z). Dirección Académica, Subdirección de Investigación (MC). Universidad de Chile, Santiago, Chile.

Servicio Médico y Dental de Alumnos (JB, CH).

Financiamiento: estudio financiado por Corporación Farmacéutica Recalcine, División Gynopharm Conflicto de interés: C. F. Recalcine no tuvo relación con el diseño del protocolo ni con el análisis de los datos. Las investigadoras no recibieron honorarios del laboratorio por concepto de este estudio.

Recibido: 21 de noviembre de 2012 Aceptado: 31 de julio de 2013

Correspondencia a: Carolina Conejero Roós cconejero@clc.cl 
Debido a la alta prevalencia, morbilidad asociada y presentación poco sintomática de la infección por $C$. trachomatis y $N$. gonorrhoeae, el Centro de Control y Prevención de Enfermedades (CDC, Atlanta, E.U.A.) ha recomendado el tamizaje anual para búsqueda de ambos patógenos en mujeres sexualmente activas bajo 26 años de edad $^{18}$, medida que en Chile existe como recomendación del Comité de Adolescencia del Ministerio de Salud ${ }^{19}$. La detección precoz y el tratamiento constituyen estrategias para reducir la duración de la infección, el riesgo de transmisión a las parejas sexuales y la aparición de complicaciones (PIP, daño tubario e infertilidad $)^{20}$.

La técnica clásica para diagnóstico de $C$. trachomatis y $N$. gonorrhoeae es el cultivo. Este método es altamente específico; sin embargo, su sensibilidad es deficiente (70$85 \%$ ) lo que limita su uso como método de tamizaje. Las técnicas recomendadas actualmente para tamizaje de $C$. trachomatis y $N$. gonorrhoeae se basan en la amplificación de ácidos nucleicos por reacción de polimerasa en cadena (RPC), método que tiene alta sensibilidad y especifici$\mathrm{dad}^{5-7}$. La muestra puede ser obtenida de endocérvix, vagina, uretra $\mathrm{u}$ orina.

Como una manera de favorecer la adherencia de las pacientes al tamizaje, en los últimos años se ha trabajado en el desarrollo de técnicas menos invasoras como la autotoma de muestra vaginal. Estudios comparativos entre el análisis de muestras obtenidas por autotoma y obtención por ginecólogo(a) han evidenciado un rendimiento similar en cuanto a la detección de $C$. trachomatis y $N$. gonorrhoeae; las muestras vaginales obtenidas por estos métodos tendrían una rendimiento superior a las muestras endocervical y de orina de primer chorro (sensibilidad por autotoma vaginal de $93 \%$, endocervix $91 \%$, orina $80,6 \%)^{21,22}$. A la fecha, no existen estudios sistemáticos que hayan comunicado la percepción y/o satisfacción de las mujeres frente a la autotoma vaginal.

El objetivo de este trabajo fue determinar la frecuencia de detección de $C$. trachomatis y $N$. gonorrhoeae por un método de autotoma de muestra vaginal y su aceptabilidad en un grupo de adolescentes y jóvenes adultas chilenas cursando estudios universitarios.

\section{Pacientes y Método}

\section{Diseño}

Estudio descriptivo, de corte transversal, realizado entre marzo y agosto del año 2011.

\section{Pacientes}

Se incluyeron mujeres de 18 a 25 años, con inicio de actividad sexual, no menstruando en el momento del examen, que fueran capaces de entender las instrucciones de la autotoma de muestra vaginal y que consultaran es- pontáneamente en la Unidad de Ginecología Adolescente de Clínica Las Condes (CLC) y la Unidad de Ginecología del Servicio de Salud Estudiantil de la Universidad de Chile (SEMDA). Se excluyeron mujeres embarazadas y aquéllas que hubieran recibido antimicrobianos en el último mes. Todas las pacientes firmaron consentimiento informado y el estudio fue aprobado por el Comité de Ética de Clínica Las Condes.

\section{Registro de datos y definición de las variables}

Se aplicó una encuesta para el registro de datos epidemiológicos e información relacionada con la actividad sexual (edad de inicio de relaciones sexuales, número de parejas sexuales, uso de preservativo, uso de método anticonceptivo), síntomas uro-ginecológicos (flujo vaginal, molestias urinarias, sangrado post coital), aceptabilidad del método de autotoma (comprensión de las instrucciones, facilidad, comodidad, preferencia de la autotoma).

\section{Toma y análisis de las muestras vaginales}

Se instruyó a las pacientes acerca de la técnica de autotoma vaginal con tórula de dacrón (GenProbe, USA). Las muestras fueron tomadas en la consulta, transportadas el mismo día en frío al laboratorio de Biología Molecular de Clínica Las Condes y mantenidas a $4^{\circ} \mathrm{C}$ hasta su procesamiento.

Luego de la extracción de ácidos nucleicos mediante método automatizado, se realizó amplificación mediada por transcripción usando el kit comercial Aptima Combo $2^{\circledR}$ (GenProbe, USA), de acuerdo a las instrucciones del fabricante. Los resultados fueron informados a cada paciente y se indicó terapia antimicrobiana en los casos positivos.

\section{Resultados}

Se ofreció participar en el estudio a 347 pacientes, de las cuales 344 aceptaron y fueron incluidas en el análisis. De éstas, 160 fueron reclutadas en CLC y 184 en SEMDA (46,5 y $53,4 \%$, respectivamente).

En la Tabla 1 se describen las características de la población y conducta sexual de las pacientes. Los promedios de edad actual y al inicio de su actividad sexual fueron 21,7 años ( $\pm 1,9$ años) y 17,9 años ( $\pm 1,9$ años), respectivamente.

No se encontraron diferencias estadísticamente significativas entre las pacientes reclutadas en ambos centros en relación a las variables edad, edad de inicio de actividad sexual y número de parejas sexuales. En el grupo reclutado en CLC se encontró una proporción significativamente mayor de pacientes sin relaciones sexuales durante el mes previo al estudio $(26,1$ y $13,1 \%$, respectivamente; p: 0,002$)$ y mayor proporción de uso de preservativo en el último mes (43 y 38\% respectivamente). 
En la Tabla 2 se presentan los datos de frecuencia de C. trachomatis y asociación de ésta con edad, conducta sexual y síntomas. La frecuencia de $C$. trachomatis en el grupo total fue de $7,9 \%$, sin diferencias significativas entre las pacientes reclutadas en ambos centros. No se encontraron muestras positivas para $N$. gonorrhoeae.

La presencia de flujo genital se asoció a un aumento de 1,5 veces en el riesgo de infección por C. trachomatis. No se encontró asociación de riesgo de infección por $C$. trachomatis para ninguna de las otras variables estudiadas (edad, número de parejas sexuales, número de relaciones sexuales en el último mes, uso de preservativo o molestias urinarias).

En la Tabla 3 se muestra la opinión de las pacientes respecto a preferencia, comodidad y facilidad de las instrucciones para la técnica de autotoma de muestra vaginal. La mayoría de las pacientes $(97,7 \%)$ consideró que las instrucciones para la autotoma eran fáciles de entender, sin diferencias entre los grupos CLC y SEMDA. Se encontró un predominio de pacientes que preferían el método de autotoma respecto a la toma por un profesional de la salud (50,9\%). Este predominio fue mayor en el grupo de CLC (55,6 y 46,7\% de preferencias, respectivamente) si bien no alcanzó significancia estadística (p: 0,100). Al determinar la comodidad de la autotoma se encontró que $87,5 \%$ del total de las pacientes se sintió cómoda al tomar la muestra, no existiendo diferencia estadísticamente significativa entre ambos grupos.

\section{Discusión}

La prevalencia de C. trachomatis encontrada en este estudio $(7,9 \%)$ se asemeja a otros reportes nacionales. Huneeus ${ }^{15}$ y cols., en un estudio con muestra de orina de primer chorro, encontraron una prevalencia de $6,9 \%$ en adolescentes y jóvenes chilenas, lo que podría explicarse por una mayor sensibilidad de las muestras obtenidas por autotoma vaginal, lo que ya ha sido descrito ${ }^{22}$. Schachter y cols., compararon la detección de C. trachomatis en muestras obtenidas de orina de primer chorro, autotoma vaginal, toma vaginal realizada por el médico clínico y toma endocervical, encontrando que la mayor sensibilidad en el tamizaje para C. trachomatis se obtiene mediante autotoma vaginal.

En relación a $N$. gonorrhoeae, en nuestro estudio no encontramos casos positivos, lo que concuerda con la disminución progresiva de esta patología en nuestro país ${ }^{23}$.

En la prevención del contagio de una ITS, es fundamental el uso de condón; sin embargo, en nuestro estudio no encontramos diferencias en su uso en el último mes, en pacientes $C$. trachomatis $(+)$ versus aquellas $C$. trachomatis (-). Esto puede explicarse por la dificultad en determinar el uso consistente de preservativo mediante una encuesta y al desconocimiento que tiene nuestra po-
Tabla 1. Características de la población y conductas sexuales

\begin{tabular}{|c|c|c|c|c|}
\hline Variable & $\begin{array}{l}\text { SEMDA } \\
\text { ( } n: 184)\end{array}$ & $\begin{array}{c}\text { CLC } \\
\text { (n: 160) }\end{array}$ & $\begin{array}{l}\text { Total } \\
\text { (n: } 344)\end{array}$ & $\mathrm{p}$ \\
\hline Promedio edad (años) (DS ) & $21,8(1,8)$ & $21,5(2,1)$ & $21,7(1,9)$ & 0,571 \\
\hline $\begin{array}{l}\text { Promedio de edad de inicio de relaciones } \\
\text { sexuales, (años) ( DS) }\end{array}$ & $17,9(1,9)$ & $18,0(1,8)$ & $17,9(1,9)$ & 0,475 \\
\hline $\begin{array}{l}\text { No parejas sexuales durante la vida (\%) } \\
1-3 \\
4-6 \\
7-9 \\
100+ \\
\text { No responde }\end{array}$ & $\begin{array}{rr}145 & (79 \%) \\
24 & (13 \%) \\
8 & (4 \%) \\
6 & (3 \%) \\
1 & (1 \%)\end{array}$ & 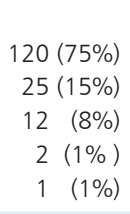 & $\begin{array}{rr}265 & (77 \%) \\
49 & (14 \%) \\
20 & (6 \%) \\
8 & (2 \%) \\
2 & (1 \%)\end{array}$ & 0,475 \\
\hline $\begin{array}{l}\text { No de relaciones sexuales en el últimos mes } \\
(\%) \\
0 \\
1-5 \\
6-10 \\
11-15\end{array}$ & $\begin{array}{c}24(13 \%) \\
97(53 \%) \\
52(29 \%) \\
10(5 \%)\end{array}$ & $\begin{array}{c}41(26 \%) \\
81(52 \%) \\
28(18 \%) \\
7 \quad(4 \%)\end{array}$ & $\begin{array}{r}65(19 \%) \\
178(52 \%) \\
80(24 \%) \\
17(5 \%)\end{array}$ & 0,008 \\
\hline
\end{tabular}

Tabla 2. Detección de Chlamydia trachomatis en muestra vaginal obtenida por autotoma y factores asociados

\begin{tabular}{|c|c|c|c|c|c|c|}
\hline \multirow[t]{2}{*}{ Variable } & \multicolumn{2}{|c|}{ SEMDA } & \multicolumn{2}{|c|}{ CLC } & \multicolumn{2}{|c|}{ Total } \\
\hline & $\mathrm{CT}(+)$ & CT (-) & CT (+) & CT (-) & $\mathrm{CT}(+)$ & CT (-) \\
\hline & $13(7 \%)$ & 171 (93\%) & $14(9 \%)$ & 146 (91\%) & $27(8 \%)$ & 317 (92\%) \\
\hline & & & & & \multicolumn{2}{|c|}{ Valor $p$} \\
\hline Edad & & & & & \multicolumn{2}{|c|}{0,87} \\
\hline n Parejas s & la vida (\% & & & & \multicolumn{2}{|c|}{0,37} \\
\hline Uso de pr & & & & & \multicolumn{2}{|c|}{0,630} \\
\hline Flujo vagir & & & & & \multicolumn{2}{|c|}{0,04} \\
\hline Molestias & & & & & \multicolumn{2}{|c|}{0,191} \\
\hline
\end{tabular}

Tabla 3. Opinión de las pacientes repsecto al método de autotoma muestra vaginal

\begin{tabular}{lrrrc} 
Variable & SEMDA & CLC & Total & p \\
Preferencia autotoma & & & & 0,001 \\
& $86(47 \%)$ & $89(56 \%)$ & $175(51 \%)$ & \\
- No prefiere & $49(27 \%)$ & $23(14 \%)$ & $72(21 \%)$ & \\
- No sabe & $39(21 \%)$ & $27(17 \%)$ & $66(19 \%)$ & \\
- Indiferente & $8(4 \%)$ & $16(10 \%)$ & $24(7 \%)$ & \\
& $2(1 \%)$ & $5(3 \%)$ & $7(2 \%)$ & \\
Comodidad & & & & 0,327 \\
- Cómoda & $158(86 \%)$ & $143(90 \%)$ & $301(88 \%)$ & \\
- Incómoda & $24(13 \%)$ & $13(8 \%)$ & $37(10 \%)$ & \\
& $2(1 \%)$ & $4(2 \%)$ & $6(2 \%)$ & \\
Instrucciones & & & & 0,068 \\
- Instrucciones fáciles & $181(99 \%)$ & $155(97 \%)$ & $336(98 \%)$ & \\
- Instrucciones no fáciles & $3(1 \%)$ & $1(1 \%)$ & $4(1 \%)$ & \\
& $0(0 \%)$ & $4(2 \%)$ & $4(1 \%)$ & \\
\hline
\end{tabular}

SEMDA: Servicio Médico de Alumnos. CLC: Clínica Las Condes. 
blación acerca del significado real del uso consistente de condón masculino, por lo que consideramos fundamental insistir en una mejor educación sexual a nuestra población, que incluya este tema.

La técnica de autotoma vaginal fue bien aceptada por las pacientes. Existe, sin embargo, un pequeño grupo que hubiera preferido la toma por personal de salud, refiriendo este grupo de pacientes, preocupación acerca de la seguridad de una muestra adecuada. La aceptación de esta técnica lo consideramos de suma importancia, ya que en población joven, asintomática, en las que el examen ginecológico con espéculo genera gran temor y ansiedad, éste podría diferirse hasta que hayan transcurrido tres años desde el inicio de la actividad sexual, o a la edad de 21 años, para la toma del PAP $^{24}$.

Dados los resultados encontrados en nuestro estudio y en otros estudios en nuestro país, pensamos que sería recomendable realizar el estudio de autotoma vaginal, en todas las mujeres consultantes en al menos los Centros de Vigilancia Centinela de ITS de nuestro país, que atiende población de mayor riesgo, y además creemos de vital importancia realizar estudios poblacionales de mayor magnitud con énfasis en población más representativa a nivel nacional, para así establecer la real prevalencia de esta infección.

Debemos destacar además que estudios del CDC, Atlanta $^{18}$, han demostrado que el costo de realizar tamizaje es menor al tratamiento de las secuelas de la infección por C. trachomatis.

Dentro de las fortalezas del estudio se puede mencionar que es el estudio transversal con más pacientes enroladas del segmento adolescentes y jóvenes, realizado hasta la fecha en nuestro país. Además, es el primero en evaluar la satisfacción de la autotoma en población de adolescentes y jóvenes chilenas, ya que no existen estudios previos con esta técnica en Chile, porque es relativamente nueva en nuestro país y aún no se utiliza en forma masiva. Nuestros hallazgos concuerdan con estudios internacionales que evalúan la satisfacción de la usuaria ${ }^{25-27}$

Dentro de las limitaciones, cabe mencionar que se trató de un grupo de pacientes seleccionadas, que consulta a un centro de salud, la mayoría con estudios universitarios, por lo que podría no ser una muestra representativa de la población general. Es posible que la prevalencia y la aceptación de la autotoma en población adolescente y joven de menores ingresos y menor nivel educacional, sean diferentes.

De acuerdo a los resultados obtenidos, es importante insistir en la pesquisa anual de $C$. trachomatis, siendo la técnica de autotoma una alternativa válida, que se complementa con la educación a la población en una sexualidad responsable y segura.
En conclusión, la prevalencia de $C$. trachomatis en la población estudiada fue similar a lo descrito internacionalmente y a los reportes nacionales. En relación a la técnica de autotoma vaginal, ésta fue bien aceptada y evitó el uso de espéculo en las pacientes. Sin embargo, algunas mujeres manifestaron ansiedad sobre la seguridad de una toma adecuada. De acuerdo a nuestros resultados, es importante insistir en la detección anual de este patógeno, siendo la técnica de autotoma una alternativa fácil, aceptada y válida.

\section{Resumen}

Introducción: Chlamydia trachomatis y Neisseria gonorrhoeae son causantes de 3 a 10\% de las infecciones de transmisión sexual en adolescentes. Las normas internacionales recomiendan su detección anual en mujeres sexualmente activas menores de 26 años. La adherencia a este tamizaje en mujeres jóvenes está limitada por el temor al examen ginecológico y alto costo del examen. Objetivo: Determinar la frecuencia de detección de $C$. trachomatis y $N$. gonorrhoeae por un método de autotoma de muestra vaginal y su aceptabilidad en un grupo de adolescentes y jóvenes adultas. Pacientes y Método: Se incluyeron mujeres de 18 a 25 años atendidas en Clínica Las Condes y el Servicio de Salud Estudiantil de la Universidad de Chile, que fueron instruidas para autotoma de muestra vaginal. Luego de dar su consentimiento, las muestras fueron estudiadas mediante reacción de polimerasa en cadena para la detección de $C$. trachomatis y $N$. gonorrhoeae. Se recopilaron datos sobre conductas sexuales y percepción de la autotoma mediante encuesta. Se determinó la relación entre estos factores y la aceptabilidad del método. Resultados: Se reclutaron 344 mujeres, con una edad promedio de 21,7 años. La detección de C. trachomatis fue de 7,9\% y no se encontró muestra positiva para $N$. gonorrhoeae. El reporte de flujo vaginal por la paciente se asoció a 1,5 veces mayor riesgo de C. trachomatis. El 98\% consideró las instrucciones de la autotoma fáciles de entender, 87,5\% se sintió cómoda al tomar la muestra. Conclusiones: La prevalencia de $C$. trachomatis en la población estudiada fue similar a lo descrito en otras series nacionales e internacionales; no se encontró $N$. gonorrhoeae en esta serie, lo que coincide con lo reportado en el extranjero. La técnica de autotoma de muestra vaginal fue bien aceptada por las pacientes; sin embargo, manifestaron ansiedad acerca de la seguridad de una toma adecuada. De acuerdo a nuestros resultados, es importante insistir en la detección anual de estos patógenos siendo la técnica de autotoma una alternativa válida. 


\section{Referencias bibliográficas}

1.- Sweet R. Treatment of acute pelvic inflammatory disease. Infect Dis Obstet Gynecol 2011; 2011: 1-13.

2.- Baraitser P, Alexander S, Sheringham J. Chlamydia trachomatis screening in young women. Curr Opin Obstet Gynecol 2011; 23 (5): 315-20.

3.- Lehtinen M, Ault K A, Lyytikainen E, Dillner J, Garland S M, Ferris D G. Future I and II study group. Chlamydia trachomatis infection and risk of cervical intraepithelial neoplasia. Sex Transm Infect 2011; 87 (5): 372-6.

4.- Fleming D T, Wasserheit J N. From epidemiological synergy to public health policy and practice, the contribution of other sexually transmitted diseases to sexual transmission of HIV infection. Sex Transm Infect 1999; 75 (1): 3-17.

5.- Miller W C, Ford C A, Morris M, Handcock M D, Schmitz J L, Hobbs M M, et al. Prevalence of chlamydial and gonococcal infections among young adults in the United States. JAMA 2004; 291 (18): 2229-36.

6.- Stein C R, Kaufman J S, Ford C A, Leone P A, Feldblum P J, Miller W C. Screening young adults for prevalent chlamydial infection in community settings. Ann Epidemiol 2008; 18 (7): 560-71.

7.- Mylonas I. Female genital Chlamydia trachomatis infection: where are we heading? Arch Gynecol Obstet 2012; Feb 19.

8.- Molano M, Weiderpass E, Posso H, Morre S A, Ronderos M, Franceschi S, et al. HPV Study Group. Prevalence and determinants of Chlamydia trachomatis infections in women from Bogota, Colombia. Sex Transm Infect 2003; 79: 474-8.

9.- Sánchez R, Ruiz-Parra A, Ostos-Ortiz O. Prevalencia de Chlamydia trachomatis detectada por reacción en cadena de la polimerasa en un grupo de mujeres jóvenes sintomáticas y asintomáticas en Bogotá, Colombia. Rev Colombiana Obstet Ginecol 2006; 57 (3): 171-81.

10.- García P J, Chávez S, Feringa B, Chiappe M, Li W, Jansen K U, et al. Reproductive tract infections in rural women from the highlands, jungle, and coastal regions of Peru. Bull World Health Organ 2004; 82: 483-7.

11.- Araújo R, Guimarães E, Alves M, Sakurai E, Domingos L, Fioravante F, et al. Prevalence and risk factors for Chlamydia trachomatis infection in adolescent females and young women in central Brazil. Eur J Clin Microbiol Infect Dis 2006; 25: 397-400.

12.- Codes J S, Cohen D A, Melo N A, Santos A B, Codes J J G, Silva Júnior J C, et al. Detecção de doenças sexualmente transmissíveis em clínicas de planejamento familiar da rede pública no Brasil. Rev Bras Ginecol Obstet 2002; 24: 1016.

13.- Arráiz N, Ginestre M, Perozo A, Castellano M, Urdaneta B y García M. Diagnóstico molecular y prevalencia de infecciones por Chlamydia trachomatis en pacientes sintomáticas y asintomáticas de una población del estado de Zulia, Venezuela. Rev Chilena Infectol 2007; 24 (1): 48-52.

14.- Martínez MA, Reid I, Arias C, Napolitano C, Sandoval J, Molina R. Prevalencia de infección cervical por Chlamydia trachomatis en mujeres de la Región Metropolitana. Rev Med Chile 2008; 136: 1294-300.

15.- Huneeus A, Pumarino M G, Schilling A, Robledo P, Bofil M. Prevalencia de Chlamydia trachomatis y Neisseria gonorrhoeae en adolescentes chilenas. Rev Med Chile 2009; 137 (12): 1569-74.

16.- Parish W, Laumann E, Cohen M, Pan S, Zheng H, Hoffman I, et al. Population-based study of Chlamydial infection in China: a hidden epidemic. JAMA 2003; 289: 1265-73.

17.- Santander E, Fish F, Salvo A, Pacheco G. Normas de manejo y tratamiento de las infecciones de transmisión sexual. Primera parte. Rev Chilena Infectol 2009; 26 (2): 174-90.

18.- Centers for Disease Control and Prevention. Sexually Transmitted Diseases Treatment Guidelines, 2010. MMWR Morbid Mortal Wkly Rep 2010; 59 (No. RR-12): 44-9.

19.- Recomendaciones Ministerio Comité Adolescencia 2009. Normas de manejo y tratamiento de infecciones de transmisión sexual (ITS). En www.minsal.gob.cl/portal/ url/item/85381414c56411a9e04001011e015920. pdf

20.- Scholes D, Stergachis A, Heidrich F E, Andrilla H, Holmes K K, Stamm W E. Prevention of pelvic inflammatory disease by screening for cervical chlamydial infection. $\mathrm{N}$ Engl J Med 1996; 34 (21): 1362-66.

21.- Jenkins W, Weis R, Campbell P, Barnes M, Barnes P, Gaydos Ch. Comparative effectiveness of two self-collected sample kit distribution systems for chlamydia screening on a university campus. Sex Transm Infect 2012; 10: 1-5.

22.- Schachter J, Mc Cormack W M, Chernesky M A, Martin D H, Van Der Pol B, Rice P A, et al. Vaginal swabs are appropriate specimens for diagnosis of genital tract infection with Chlamydia trachomatis. J Clin Microbiol 2003; 41 (8): 3784-9.

23.- Boletín Electrónico Mensual de Vigilancia Epidemiológica, Gobierno de Chile Gonorrea Situación epidemiológica semanas 1-19 año 2012; número 104. Disponible en http://epi. minsal.cl

24.- ACOG Practice Bulletin. Cervical citology screening. Obstet Gynecol 2003; 102 (2): 417-27.

25.- Chernesky M A, Hook E W, Martin D H, Lane J, Johnson R, Jordan J A, et al. Women find it easy and prefer to collect their own vaginal swabs to diagnose Chlamydia trachomatis or Neisseria gonorrhoeae infections. Sex Transm Dis 2005; 32 (12): 729-33.

26.- Richardson E, Sellors J W, Mackinnon S, Woodcox V, Howard M, Jang D, et al. Prevalence of Chlamydia trachomatis infections and specimen collection preference among women, using self collected vaginal swabs in community settings. Sex Transm Dis 2003; 30 (12): 880-5.

27.- Tebb K P, Paukku M H, Pai-Dhungat M R, Gyamfi A A, Shafer MA. Home STI testing: the adolescent female's opinion. J Adolesc Health 2004; 35 (6): 462-7. 